\title{
Outcomes of Subdural Hemorrhage in the Geriatric Population Older Than 80 Years of Age
}

\author{
Ji Hyun Oh, Hyun Seok Lee, Yoo Sung Jeon, Young II Chun, Joon Cho, Chang Taek Moon, Young-Cho Koh \\ Department of Neurosurgery, Konkuk University Medical Center, Konkuk University College of Medicine, Seoul, Korea
}

Objective: Chronic subdural hematoma (CSDH) is a common neurosurgical disorder, and its incidence increases with age. The aim of this study was to compare the outcomes of elderly and younger patients with CSDH who underwent burr-hole craniotomy.

Methods: We retrospectively reviewed the records of 79 patients with unilateral CSDH who underwent burr-hole craniotomy. We compared the data of 28 patients older than 80 years and 51 patients younger than 80 years. Each patient's symptoms, radiologic data, complications, and hospitalization duration were analyzed. Additionally, we evaluated each patient's modified Rankin Scale (mRS) score to assess the clinical outcomes.

Results: The mean ages of the older and younger patients were 84.5 and 68.6 years, respectively. Older patients were more likely to develop postoperative complications and remained hospitalized for longer because of complications. Both the preoperative maximal hematoma thickness and the pre- and postoperative difference in thickness were greater in the older group. Preoperatively, the younger group contained a higher percentage of patients with an mRS score of 0-2. Six months postoperatively, both groups had similar percentages of patients with an mRS score of 0-2.

Conclusion: Our findings showed that larger hematoma volumes were removed from older group after surgery during similar periods. However, the significantly higher incidence of general complications in the older group led to significantly longer hospitalization. However, the good treatment outcomes and prognosis suggest that burr-hole craniotomy can be an effective treatment even for elderly patients if clinicians remain aware of the prevention and treatment of postoperative complications.

Key Words: Aged, Chronic, Hematoma, Postoperative complications, Subdural, Trephining

\section{INTRODUCTION}

A chronic subdural hematoma (CSDH) is an encapsulated accumulation of blood between the dura mater and arachnoid mater $^{2,12)}$. CSDH is a common neurosurgical disease ${ }^{4,5,11,13,15,16}$, and its incidence increases with age ${ }^{8)}$.

Numerous original studies and reviews have focused on the surgical techniques for CSDH and factors associated with recurrence, however, few studies have focused on CSDH in elderly patients ${ }^{4,15,16}$, despite the increased incidence with age. This is particularly concerning because the incidence of CSDH

Corresponding author: Young II Chun, MD, PhD Department of Neurosurgery, Konkuk University Medical Center, Konkuk University College of Medicine, 120-1, Neungdong-ro, Gwangjin-gu, Seoul 05030, Korea Tel: +82-2-2030-7357, Fax: +82-2-2030-7359 E-mail: youngil.chun@gmail.com among elderly patients continues to increase as the world population becomes older ${ }^{1}$. The overall incidence of CSDH is approximately $20.6 / 100,000$ per year in the general population and 127.1/100,000 per year in patients older than 80 years, who have been found to comprise one-third of the total CSDH population ${ }^{3,13)}$. The peak age of CSDH occurrence has also increased. CSDH occurrence peaked in the 50s, 60s, and 70s in the 1970s, 1980s, and 1990s, respectively. In 2018, Hiroyuki et al. reported that the 80 s was the most common age for developing $\mathrm{CSDH}^{14,15)}$. Although the definition of "elderly" usually includes individuals older than 65 years, the world population progressively becomes older. Therefore, it is considered that studies are now required on "older aged" patients. The proportion of patients with CSDH older than 80 years is high and is expected to increase further. The aim of this study was to compare the outcomes of patients with CSDH older and younger than 80 years treated with burr-hole craniotomy surgery. 


\section{MATERIALS AND METHODS}

\section{Patients and Method}

We retrospectively reviewed the medical records of $79 \mathrm{pa}-$ tients with unilateral CSDH who underwent burr-hole craniotomy surgery between 2014 and 2018. Within this patient sample, we compared the clinical details and outcomes of 28 patients older than 80 years (older group) with those of 51 patients younger than 80 years (younger group). Patients with bilateral CSDH were excluded from this study because in such cases, the maximal hematoma thickness and midline shift thickness can interfere with each other.

\section{Treatment}

In each case, the radiologic diagnosis of $\mathrm{CSDH}$ was confirmed by a computed tomography (CT) scan. Each patient's history of anticoagulation and head trauma, the symptoms at the time of the hospital visit, radiologic data, including the midline shift thickness and maximal thickness of the hematoma, and postoperative complications were analyzed. The patients' conditions were assessed using the modified Rankin Scale (mRS) scores recorded before and immediately after surgery and 1 and 6 months after discharge.

The maximal midline shift and hematoma thickness were measured as depicted in Figure 1. The midline was measured as the line between the anterior and posterior attachments of the falx to the inner skull table. The midline shift was measured as the perpendicular distance between the midline and the displaced septum pellucidum. The degree of midline shift was defined as the ratio of the midline shift to the width of the cerebral hemisphere (i.e., the perpendicular distance between the midline and the inner skull table at the most convex point). The maximal hematoma thickness was the thickest width of the hematoma on brain CT, while the degree of maximal hematoma thickness was defined as the ratio of the hematoma thickness to the width of the hemisphere.

The indications for surgery included symptomatic CSDH. A single burr-hole craniotomy was performed under general anesthesia, followed by the placement of a subdural drain. The subdural drain was removed when the drainage volume decreased to $<10 \mathrm{cc}$ within 24 hours.

\section{Statistical Analysis}

Re-operation was defined as a repeated surgery for an ipsi- lateral recurrent $\mathrm{CSDH}$ within 1 year. We used the chi-square test and Fisher's exact test to analyze the data using SPSS (IBM Corp., Armonk, NY, USA) and values $\mathrm{p}<0.05$ were considered significant.

\section{RESULTS}

The baseline data of the 79 patients treated for CSDH are described in Table 1.

The patients' mean ages were $84.5 \pm 3.8$ (range: $80-96$ ) and $68.6 \pm 11.1$ (19-79) years in the older and younger groups, respectively. Although the older group had a slightly high anticoagulation rate and percentage of patients with a trauma history, there was no statistically significant difference in these variables between the groups.

In both groups, the main symptom was hemiparesis, followed by headaches in the younger group and an altered mental status in the older group. The rate of neurologic symptoms was slightly but non-significantly higher in the older group, whereas the rate of general symptoms was higher in the younger group $(p=0.035)$. Patients in the younger group were more likely to present with headache and signs of increased intracranial pressure. Elderly patients, more frequently presented with hemiparesis and hemispheric symptoms.

The clinical outcomes of the two groups are summarized in Table 2.

The rates of re-operation were $9.8 \%$ in the younger group and $3.6 \%$ in the older group, and this difference was not statistically significant.

Overall postoperative complications were more common in the older group, which had a complication rate of 53.1\% versus $20 \%$ in the younger group $(\mathrm{p}=0.004)$. The rate of gen-

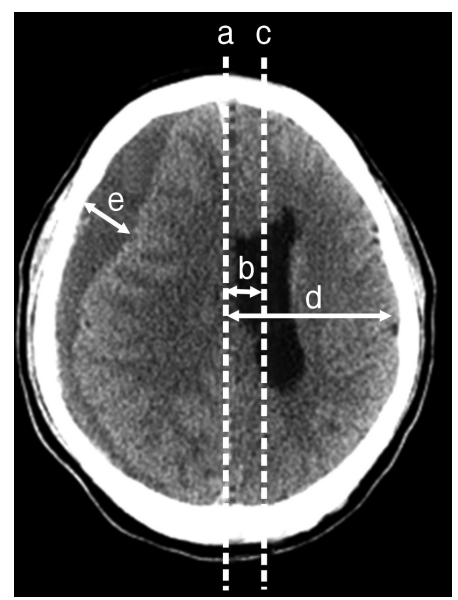

Fig. 1. Measurement of the maximal hematoma thickness and midline shift on a representative brain computed tomography image. a: Mid- line, b: Midline shift, c: Shifted septum pellucidum, $\mathrm{d}$ : Width of the cerebral hemisphere, e: Maximal thickness of the hematoma. 
eral complications including pneumonia, urinary tract infections, and wound infections was significantly higher in the older group ( $46.4 \%$ vs. $11.8 \%$ in younger patients, $\mathrm{p}=0.001$ ), whereas the rate of neurologic complications including seizures and delirium was not significantly different between the two groups. In the older group, pneumonia (18.8\%) was the most common complication, followed by urinary tract infections (12.5\%).

The mean duration of drainage was 4.2 days in both groups, and the length of hospitalization after surgery was 9.4 days in the younger group and 14.5 days in the older group. This difference in hospitalization length was attributed to the hig-

Table 1. Baseline characteristics of patients

\begin{tabular}{lccc}
\hline \hline & $<80$ Years of age $(\mathrm{n}=51)$ & $\geq 80$ Years of age $(\mathrm{n}=28)$ & p-value \\
\hline Mean age, years (range) & $68.6(19-79)$ & $84.5(80-96)$ & \\
Sex (male : female) & $2: 1$ & $1.2: 1$ & \\
Anti-coagulation (\%) & $16(31.4)$ & $10(35.7)$ & 0.694 \\
Trauma history (\%) & $28(54.9)$ & $18(64.3)$ & 0.419 \\
Symptoms (\%) & & & \\
Neurologic symptoms (\%) & $34(66.7)$ & $22(78.6)$ & 0.265 \\
Hemiparesis & $26(35.6)$ & $17(47.2)$ & \\
Speech disturbance & $7(9.6)$ & $4(11.1)$ & \\
Mental change & $2(2.7)$ & $6(16.7)$ & \\
Amnesia & $2(2.7)$ & $0(0)$ & \\
Diplopia & $1(1.4)$ & $0(0)$ & \\
General symptoms (\%) & $29(56.9)$ & $9(32.1)$ & \\
Headache & $24(32.9)$ & $3(8.3)$ & \\
Dizziness & $6(8.2)$ & $5(13.9)$ & \\
Nausea/Vomiting & $3(4.1)$ & $0(0)$ & \\
General weakness & $1(1.4)$ & $0(0)$ & \\
Urinary incontinence & $1(1.4)$ & $1(2.8)$ & \\
\hline
\end{tabular}

Table 2. Comparison of the surgical results

\begin{tabular}{lccc}
\hline \hline & $<80$ Years of age $(\mathrm{n}=51)$ & $\geq 80$ Years of age $(\mathrm{n}=28)$ & p-value \\
\hline Re-operation (\%) & $5(9.8)$ & $1(3.6)$ & 0.317 \\
Post-operative complications (\%) & & & \\
$\quad$ None & $44(80)$ & $15(46.9)$ & 0.004 \\
Neurologic complications (\%) & $5(9.8)$ & $2(7.1)$ & 0.691 \\
Seizure & $3(5.5)$ & $0(0)$ & \\
Delirium & $2(3.6)$ & $2(6.3)$ & 0.001 \\
General complications (\%) & $6(11.8)$ & $13(46.4)$ & \\
Pneumonia & $3(5.5)$ & $6(18.8)$ & \\
UTI (Urinary tract infection) & $2(3.6)$ & $4(12.5)$ & \\
Wound infection & $1(1.8)$ & $0(0)$ & \\
Voiding difficulty & $0(0)$ & $2(6.3)$ & $1(3.1)$ \\
Arrhythmia & $0(0)$ & $1(3.1)$ & \\
Deep vein thrombosis & $0(0)$ & $1(3.1)$ & \\
Colitis & $0(0)$ & $1(3.1)$ & 0.874 \\
Phlebitis & $0(0)$ & $4.2(1-10)$ & \\
Duration of drainage, mean, days (range) & $4.2(3-9)$ & $14.5(3-133)$ & 0.327 \\
Hospital stay after operation, mean days (range) & $9.4(4-32)$ & & \\
\hline
\end{tabular}




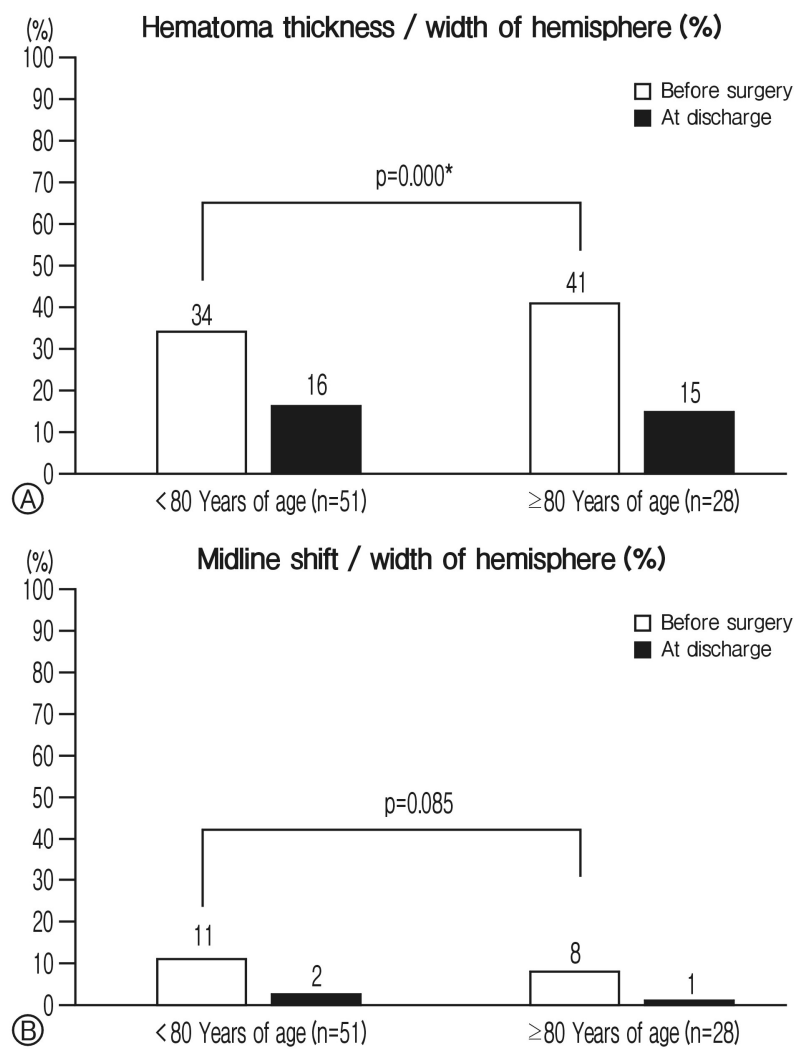

Fig. 2. Comparison of the mass effect. (A) Ratio of maximal hematoma thickness to the width of the cerebral hemisphere before and after surgery. (B) Ratio of the midline shift to the width of the cerebral hemisphere before and after surgery. her rate of postoperative complications in the older group.

A comparison of the mass effect in both groups is summarized in Figure 2.

The mean ratio of maximal hematoma thickness to the width of the cerebral hemisphere before surgery was higher in the older group ( $41 \%$ vs. $34 \%$ for younger patients, $\mathrm{p}<0.000)$. The mean difference in this ratio from before to after surgery was also significantly higher in the older group $(\mathrm{p}<0.000)$.

Preoperatively, the mean ratio of the midline shift to width of the hemisphere was $11 \%$ in the younger group and $8 \%$ in the older group, whereas the corresponding values at the time of discharge ( $2 \%$ and $1 \%$, respectively) were not significantly different. Larger hematoma volumes were removed from the elderly patients, despite similar drainage periods between the groups. These results suggest that burr-hole craniotomy surgery effectively removed the hematoma in the older group.

The patients' $\mathrm{mRS}$ scores before and after surgery and 1 and 6 months after discharge are described in Table 3.

Before surgery, the percentages of patients with an mRS score of $0-2$ were $64.7 \%$ and $42.8 \%$ in the younger and older groups, respectively. In the older group, most patients (53.6\%) had an mRS score of 3-4 before surgery. In both groups, the largest proportion of patients had an mRS score 0-2 of at discharge, but this proportion was higher in the younger group because the older group had a higher complication rate. Al-

Table 3. Comparison of clinical outcomes

\begin{tabular}{|c|c|c|c|}
\hline mRS score & $<80$ Years of age $(n=51)$ & $\geq 80$ Years of Age $(n=28)$ & p-value \\
\hline Before surgery & & & 0.231 \\
\hline $0-2$ & $33(64.7)$ & $12(42.8)$ & \\
\hline $3-4$ & $17(33.3)$ & $15(53.6)$ & \\
\hline $5-6$ & $1(2)$ & $1(3.6)$ & \\
\hline At discharge & & & 0.243 \\
\hline $0-2$ & $48(94.1)$ & $24(85.7)$ & \\
\hline $3-4$ & $3(5.9)$ & $4(14.3)$ & \\
\hline $5-6$ & $0(0)$ & $0(0)$ & \\
\hline One month after discharge & & & 0.142 \\
\hline $0-2$ & $50(98)$ & $25(89.3)$ & \\
\hline $3-4$ & $1(2)$ & $3(10.7)$ & \\
\hline $5-6$ & $0(0)$ & $0(0)$ & \\
\hline Six months after discharge & & & 0.057 \\
\hline $0-2$ & $50(98)$ & $26(92.9)$ & \\
\hline $3-4$ & $1(2)$ & $2(7.1)$ & \\
\hline $5-6$ & $0(0)$ & $0(0)$ & \\
\hline
\end{tabular}

mRS: Modified Rankin Scale. 
though the mRS score improved at a slower rate in the older than in the younger group, the values in the two groups gradually became similar. At 6 months after discharge, the percentages of patients with an mRS score of 0-2 were $98 \%$ in the younger group and $92.9 \%$ in the older group. Therefore, from a long-term perspective, the results after burr-hole craniotomy surgery were similar in patients older and younger than 80 years.

\section{DISCUSSION}

As noted in the Introduction, $\mathrm{CSDH}$ is a common neurosurgical disorder, especially in the elderly ${ }^{4,5,13)}$. Age-related increases in cerebral atrophy and venous fragility are the major factors predisposing elderly individuals to $\mathrm{CSDH}^{2}$. During the aging process, the brain volume decreases, leading to an increase in the space between the brain and the skull ${ }^{\text {}}$. Consequently, the bridging veins are stretched and become more vulnerable to trauma ${ }^{2)}$.

Studies on the surgical treatment of elderly patients with CSDH have yielded diverse findings. For example, Sadaharu et al. reported that surgery for CSDH is safe even in patients older than 90 years $^{13)}$. Mauro et al. reported similar complication and recovery rates for patients older and younger than 90 years $^{5)}$. In contrast, Shinya et al. reported that patients older than 80 years were likely to experience a recurrence and required a longer time to be cured ${ }^{16}$. Similarly, Karibe et al. compared patients who were older and younger than 70 years and found that the older patients had significantly worse outcomes ${ }^{\rceil}$. Ooba et al. reported that patents older than 80 years exhibited mitigated postoperative improvements, compared to younger patients ${ }^{10)}$.

In our study, the main symptom in both age groups was hemiparesis. This symptom occurred significantly more frequently than other symptoms in the older group, and the rate of neurologic symptoms was higher than that of general symptoms compared to patients younger than 80 years. Hemiparesis is attributed to the direct pressure inflicted by the hematoma being on the cerebral hemisphere ${ }^{2)}$. Younger groups more often present with signs of increased intracranial pressure, while older groups more often present with hemispheric symptoms. Younger groups have a lower degree of brain atrophy than older groups, and therefore, a small amount of hematoma may increase the intracranial pressure more rapidly in younger groups ${ }^{6}$. The more severe brain atrophy in the elderly patients increases the space between the brain and the skull ${ }^{5)}$, leading to thicker hematomas in this group prior to surgery. Another difference can be observed between younger and older groups. The midline shift is less severe in older patients, whereas younger patients have a greater midline shift even at the same hematoma volume due to a lower degree of brain atrophy and smaller amount of space in the skull. Older patients begin to manifest symptoms when the amount of hematoma significantly increase, which is considered to cause hemiparesis with direct pressure on the hemisphere.

The differences in hematoma thickness before and after surgery were significantly higher in the older group, despite both groups having a similar drainage period. Therefore, burr-hole craniotomy surgery appeared to effectively remove the hematoma in the older group. The postoperative outcomes of the two groups were similar, and CT revealed no significant differences in the maximal hematoma thickness and midline shifting thickness at discharge.

Regarding the postoperative outcomes, it is important to determine the extent of persistent disability and the possibility that an elderly person can return to normal activities of daily living. Therefore, we assessed the mRS score of all patients before and after surgery. However, these scores were similar at 6 months after discharge, suggesting that from a long-term perspective, there was no significant difference in the clinical outcomes between the two groups.

However, the incidence of postoperative medical complications was significantly higher in patients older than 80 years, and this was considered to explain the longer postoperative hospitalization length in this group relative to the younger one, despite the similar subdural drain maintenance period. These complications required the use of medications such as antibiotics and other treatments, which deteriorated the general condition of the elderly patients and increased the hospital costs. At discharge, patients with an mRS score of 0-2 accounted for the largest percentage in both groups, but particularly among younger patients, due to the high complication rate in elderly patients. Mauro et al. reported an association between a careful preoperative evaluation of a patient's general condition and a low perioperative complication rate ${ }^{5)}$. Shaikh et al. reported that a mini-craniotomy under local anesthesia (vs. general anesthesia) reduced the risk of postoperative complications and the hospitalization length in patients with $\mathrm{CSDH}^{9}$. Satoshi et al. reported that elderly patients are vulnerable to postoperative complications and observed that the early mobilization of patients with $\mathrm{CSDH}$ after burr-hole surgery prevented the incidence of postoperative complications, such as 
pneumonia and urinary tract infection ${ }^{8)}$.

The data suggest that burr-hole craniotomy surgery can yield a good prognosis, regardless of the patient's age, as long as clinicians maintain a greater awareness of the patient's general status before surgery and consider the prevention and treatment of medical complications after surgery. Still, further studies on postoperative complications in elderly patients are needed.

\section{CONCLUSION}

We demonstrated the safety and efficacy of burr-hole craniotomy surgery for the treatment of CSDH in diverse age groups, although we note that postoperative complications were more frequently observed in patients older than 80 years. Careful attention to the prevention and treatment of potential postoperative complications should allow safe and complete treatment of $\mathrm{CSDH}$ in elderly patients.

Conflicts of Interest: The authors do not report any potential conflicts of interest relevant to this article.

\section{REFERENCES}

1. Adhiyaman V, Asghar M, Ganeshram KN, Bhowmick BK: Chronic subdural haematoma in the elderly. Postgrad Med J 78:71-75, 2002

2. Asghar M, Adhiyaman V, Greenway MW, Bhowmick BK, Bates A: Chronic subdural haematoma in the elderly - A North Wales experience. J R Soc Med 95:290-292, 2002

3. Balser D, Farooq S, Mehmood T, Reyes M, Samadani U: Actual and projected incidence rates for chronic subdural hematomas in united states veterans administration and civilian populations. J Neurosurg 123:1209-1215, 2015

4. Borger V, Vatter H, Oszvald A, Marquardt G, Seifert V, Guresir E: Chronic subdural haematoma in elderly patients: A retrospective analysis of 322 patients between the ages of 65-94 years. Acta Neurochir (Wien) 154:1549-1554, 2012

5. Dobran M, Marini A, Nasi D, Liverotti V, Benigni R, Costanza $\mathrm{MD}$, et al.: Clinical outcome of patients over 90 years of age treated for chronic subdural hematoma. J Korean Neurosurg Soc, 2019

6. Fogelholm R, Heiskanen O, Waltimo O: Chronic subdural hematoma in adults. Influence of patient's age on symptoms, signs, and thickness of hematoma. J Neurosurg 42:43-46, 1975

7. Karibe H, Kameyama M, Kawase M, Hirano T, Kawaguchi T, Tominaga T: Epidemiology of chronic subdural hematomas. No Shinkei Geka 39:1149-1153, 2011

8. Kurabe S, Ozawa T, Watanabe T, Aiba T: Efficacy and safety of postoperative early mobilization for chronic subdural hematoma in elderly patients. Acta Neurochir (Wien) 152:11711174,2010

9. Mahmood SD, Waqas M, Baig MZ, Darbar A: Mini-craniotomy under local anesthesia for chronic subdural hematoma: An effective choice for elderly patients and for patients in a resource-strained environment. World Neurosurg 106:676679, 2017

10. Ooba S, Shiomi N, Shigemori M: Clinical features and surgical results of chronic subdural hematoma in the extremely aged patients. No Shinkei Geka 34:273-278, 2006

11. Seizeur R, Abed-Rabbo F, Obaid S, Saliou P, Simon A, Dam Hieu P, et al.: Chronic subdural haematomas in elderly population. Neurosurgical aspects and focus on the single-burr hole technique performed under assisted local anaesthesia. $\mathrm{Br} \mathrm{J}$ Neurosurg 31:258-261, 2017

12. Szczygielski J, Gund SM, Schwerdtfeger K, Steudel WI, Oertel $\mathrm{J}$ : Factors affecting outcome in treatment of chronic subdural hematoma in ICU patients: Impact of anticoagulation. World Neurosurg 92:426-433, 2016

13. Tabuchi S, Kadowaki M: Chronic subdural hematoma in patients over 90 years old in a super-aged society. J Clin Med Res 6:379-383, 2014

14. Toi H, Kinoshita K, Hirai S, Takai H, Hara K, Matsushita $\mathrm{N}$, et al.: Present epidemiology of chronic subdural hematoma in Japan: Analysis of 63,358 cases recorded in a national administrative database. J Neurosurg 128:222-228, 2018

15. Uno M, Toi H, Hirai S: Chronic subdural hematoma in elderly patients: Is this disease benign? Neurol Med Chir (Tokyo) 57:402-409, 2017

16. Watanabe S, Kato N, Sato M, Aiyama H, Fujiwara Y, Goto $\mathrm{M}$, et al.: Treatment outcomes of burr-hole surgery for chronic subdural hematoma in the elderly living beyond life expectancy: A study comparing cure, recurrence, and complications in patients aged $>/=80$ years versus $</=79$ years. World Neurosurg 132:e812-e819, 2019 